\title{
Seletividade de Formulações de Glyphosate a Trichogramma pretiosum (HYMENOPTERA: TRICHOGRAMMATIDAE) ${ }^{1}$
}

\author{
Side-Effects of Glyphosate Formulations on Trichogramma pretiosum (Hymenoptera: \\ Trichogrammatidae)
}
GIOLO, F.P. ${ }^{2}$, GRÜTZMACHER, A.D. ${ }^{3}$, PROCÓPIO, S.O. ${ }^{3}$, MANZONI, C.G. ${ }^{2}$, LIMA, C.A.B. ${ }^{4}$ e NÖRNBERG, S.D. ${ }^{5}$

\begin{abstract}
RESUMO - O estudo foi conduzido para avaliar a seletividade de oito formulações de glyphosate (Roundup ${ }^{\circledR}$, Roundup ${ }^{\circledR}$ WG, Roundup ${ }^{\circledR}$ Transorb, Polaris ${ }^{\circledR}$, Gliz ${ }^{\circledR} 480$ CS, Glifosato Nortox $^{\circledR}$, Glifosato 480 Agripec $^{\circledR}$ e Zapp ${ }^{\circledR}$ Qi) registradas no Brasil sobre o parasitóide de ovos Trichogramma pretiosum (Hym., Trichogrammatidae). Os experimentos consistiram na exposição de adultos do parasitóide a resíduos secos das formulações pulverizadas sobre placas de vidro, na concentração de $14,4 \mathrm{mg} \mathrm{L}^{-1}$ de equivalente ácido de glyphosate. O tratamento controle recebeu água destilada. Ovos do hospedeiro alternativo Anagasta kuehniella (Lep., Pyralidae) foram ofertados para parasitismo. A capacidade de parasitismo por fêmea de T. pretiosum e a redução na capacidade, comparada com o tratamento controle, foram utilizadas para estimar a toxicidade do produto. As formulações foram classificadas em quatro categorias, conforme a redução no parasitismo: inócuo $(<30 \%)$; levemente nocivo (30-79\%); moderadamente nocivo (80-99\%); e nocivo $(>99 \%)$. Os produtos fitossanitários afetaram diferentemente a capacidade de parasitismo de $T$. pretiosum. A seletividade das formulações de glyphosate foi dependente do tipo de sal. Aquelas à base de sal potássico $\left(\right.$ Zapp $^{\circledR}$ Qi) e de sal de amônio (Roundup ${ }^{\circledR}$ WG) foram levemente nocivas a adultos do parasitóide. As demais à base de sal isopropilamina (Roundup ${ }^{\circledR}$, Polaris $^{\circledR}$, Gliz ${ }^{\circledR} 480$ CS, Glifosato Nortox ${ }^{\circledR}$, Glifosato 480 Agripec $^{\circledR}$ e Roundup ${ }^{\circledR}$ Transorb) foram moderadamente nocivas a adultos de $T$. pretiosum.
\end{abstract}

Palavras-chave: parasitóide de ovos, herbicida, controle biológico.

ABSTRACT - A study was carried out in the laboratory to investigate the side-effects reported in Brazil of eight glyphosate formulations (Roundup ${ }^{\circledR}$, Roundup ${ }^{\circledR} W G$, Roundup ${ }^{\circledR}$ Transorb, Polaris ${ }^{\circledR}$, Gliz $^{\circledR} 480$ CS, Glifosato Nortox ${ }^{\circledR}$, Glifosato 480 Agripec $^{\circledR}$ and Zapp ${ }^{\circledR}$ Qi) on the egg parasitoid Trichogramma pretiosum (Hym., Trichogrammatidae). The experiments were carried out by exposing the adult parasitoids to fresh pesticide residues applied on glass plates at a concentration of $14.4 \mathrm{mg} \mathrm{L}^{-1}$ of acid equivalent of glyphosate. The control group was treated with distilled water. Eggs of the alternative host Anagasta kuehniella (Lep., Pyralidae) were offered to parasitism. The capacity of parasitism per $\boldsymbol{T}$. pretiosum adult female and the reduction in capacity of parasitism compared with the control group were used to estimate toxicity of the product. The formulations were classified into four categories, according to parasitism reduction: 1, harmless (<30\%); 2, slightly harmful (30-79\%); 3, moderately harmful (80-99\%); 4, harmful (>99\%). The results showed that the side-effects of the glyphosate formulations were dependent on type of salt and that formulations with potassic salt (Zapp ${ }^{\circledR}$ Qi) and ammonium salt (Roundup ${ }^{\circledR} W G$ ) were slightly harmful to T. pretiosum adults. The remaining formulations isopropylamine salt (Roundup ${ }^{\mathbb{B}}$, Roundup Transorb ${ }^{\circledR}$, Polaris ${ }^{\circledR}$, Gliz ${ }^{\circledR} 480$ SC, Glifosato Nortox ${ }^{\circledR}$, Glifosato 480 Agripec $^{\circledR}$ ) were moderately harmful to $T$. pretiosum adults.

Key words: egg parasitoids, herbicide, biological control.

Recebido para publicação em 31.3.2005 e na forma revisada em 5.9.2005.

Eng.-Agr., Doutorando do PPGFs, Dep. de Fitossanidade da Universidade Federal de Pelotas - FAEM/UFPel, Campus Universitário, Caixa Postal 354 96010-000 Pelotas-RS, < fgiolo.faem@ufpel.tche.br>; ${ }^{3}$ Eng.-Agr., Dr., Prof. do Dep. de Fitossanidade - FAEM/UFPel, Bolsista de Produtividade em Pesquisa do CNPq; ${ }^{4}$ Bióloga, Bolsista de Apoio Técnico Nível Superior CNPq, Dep. de Fitossanidade - FAEM/UFPel; 5 Acadêmico de Agronomia, BIC FAPERGS, Dep. de Fitossanidade - FAEM/UFPel. 


\section{INTRODUÇÃO}

O glyphosate é o herbicida de maior participação no mercado mundial, caracterizandose pelo amplo espectro de ação no controle das plantas daninhas. Apesar de existirem mais de 90 marcas comerciais de glifosato no mundo (Heap, 1997), todas elas apresentam o mesmo mecanismo de ação, independentemente dos sais utilizados (Hartzler, 2001). No Brasil, esse herbicida é formulado com diferentes sais, como o sal potássico, sal de isopropilamina e sal de amônio.

A importância dos herbicidas formulados com glyphosate tem aumentado nos últimos anos, devido ao incremento na semeadura de culturas resistentes à molécula do glifosato (Shaner, 2000).

Embora existam informações na literatura sobre as propriedades físicas, químicas e toxicológicas do glyphosate (Amarante Júnior et al., 2002), pouco se conhece a respeito da toxicidade das formulações comerciais sobre insetos benéficos, restringindo-se ao herbicida Roundup ${ }^{\circledR}$ (Hassan et al., 1988).

Conforme o "Working Group Pesticides and Beneficial Arthropods" of the "International Organization for Biological Control of Noxious Animals and Plants (IOBC), West Palaearctic Regional Section (WPRS)", os estudos de seletividade a organismos não-alvo devem ser conduzidos com as formulações comerciais (Hassan et al., 2000), pois sabe-se que alguns adjuvantes - em especial surfatantes, no caso de herbicidas - reduzem a tensão superficial e facilitam a penetração do herbicida.

Representantes da familia Trichogrammatidae, principalmente do gênero Trichogramma, são um dos grupos mais estudados e utilizados atualmente no mundo e também no Brasil (Parra \& Zucchi, 2004) para controle de insetos-praga nos mais variados ambientes agrícolas. Das 14 espécies de Trichogramma constatadas no Brasil, Trichogramma pretiosum é a que apresenta ampla distribuição geográfica, além de ser a mais polífaga, tendo sido relatada em 18 diferentes hospedeiros e 13 culturas (Zucchi \& Monteiro, 1997).

Considerando a ampla utilização do glyphosate em todo o mundo e o registro dessa molécula para a maioria dos cultivos agrícolas no Brasil, o objetivo do presente trabalho foi avaliar a seletividade de oito diferentes formulações comerciais de glyphosate a adultos do parasitóide de ovos $T$. pretiosum, utilizando-se das linhas gerais normalizadas pela IOBC/ WPRS.

\section{MATERIAL E MÉTODOS}

O trabalho consistiu na aplicação das metodologias laboratoriais padronizadas pela IOBC/WPRS, conforme Hassan et al. (2000) e Hassan \& Abdelgader (2001), e foi conduzido nos laboratórios de Biologia de Insetos, Controle Biológico e de Pesticidas e Drogas do Departamento de Fitossanidade (DFs), pertencente à Faculdade de Agronomia "Eliseu Maciel" (FAEM) da Universidade Federal de Pelotas (UFPel), em Pelotas-RS.

Devido à capacidade operacional do sistema instalado, os testes de seletividade foram divididos em dois experimentos, sendo no experimento I avaliados os herbicidas Zapp ${ }^{\circledR}$ Qi, Roundup ${ }^{\circledR}$ WG, Roundup ${ }^{\circledR}$ e Polaris ${ }^{\circledR}$ e, no experimento II, Gliz 480 CS, Glifosato Nortox ${ }^{\circledR}$, Glifosato 480 Agripec $^{\circledR}$ e Roundup ${ }^{\circledR}$ Transorb. Todas as formulações foram pulverizadas na concentração de $14,4 \mathrm{mg} \mathrm{L}^{-1}$ de equivalente ácido de glyphosate, correspondente à dose de 2,88 $\mathrm{kg} \mathrm{ha}^{-1}$ do herbicida. O inseticida Dipterex $^{\circledR} 500$ foi utilizado como testemunha positiva, por ser reconhecidamente nocivo a adultos do parasitóide (Grützmacher et al., 2004), enquanto a testemunha negativa foi constituída por água destilada.

As pulverizações foram feitas com pulverizadores manuais com capacidade de $580 \mathrm{~mL}$, da marca Guarany ${ }^{\circledR}$, que proporcionaram depósito de calda entre 1, 5 e 2,0 $\mathrm{mg} \mathrm{cm}^{-2}$, aferido mediante balança eletrônica de precisão.

Os parasitóides $T$. pretiosum utilizados nos experimentos são oriundos de criação em câmaras climatizadas (temperatura de $25 \pm 1{ }^{\circ} \mathrm{C}$, umidade relativa de $70 \pm 10 \%$ e fotofase de 14 horas), utilizando-se ovos inviabilizados em lâmpada germicida (Stein \& Parra, 1987) do hospedeiro alternativo Anagasta kuehniella, criado conforme metodologia descrita por Parra (1997).

Os testes de toxicidade inicial foram conduzidos em laboratório, expondo-se adultos 
(estágio mais sensivel) de $T$. pretiosum a resíduos secos dos herbicidas pulverizados sobre placas de vidro de $2 \mathrm{~mm}(13 \times 13 \mathrm{~cm})$. Essas placas permaneceram à sombra por cerca de três horas após a pulverização para secagem da calda, formando uma película seca do produto-teste.

Gaiolas de exposição foram confeccionadas utilizando-se duas placas de vidro contendo o produto-teste, servindo como fundo e cobertura, e uma moldura de alumínio composta por quatro laterais $(13 \mathrm{~cm}$ de comprimento x $1,5 \mathrm{~cm}$ de altura $\times 1 \mathrm{~cm}$ de largura), que foram fixas através de presilhas. Em três laterais da moldura de aluminio havia seis orificios para ventilação (diâmetro aproximado de $1 \mathrm{~cm}$ ). No quarto lado havia dois orifícios: o maior $(3,5 \times 1 \mathrm{~cm})$ foi utilizado para introdução de ovos do hospedeiro A. kuehniella a serem parasitados e como alimento ( $3 \mathrm{~g}$ de gelatina, $100 \mathrm{~mL}$ de água e $200 \mathrm{~g}$ de mel) aos insetos em teste; o menor (diâmetro aproximado de $1 \mathrm{~cm}$ ) serviu para inserção dos tubos de emergência com os indivíduos a serem testados.

Cada tubo de emergência foi constituído por uma ampola de vidro transparente de $120 \mathrm{~mm}$ de comprimento por $20 \mathrm{~mm}$ de diâmetro em uma das extremidades e $7 \mathrm{~mm}$ na outra, contendo no seu interior um círculo de $1 \mathrm{~cm}$ de diâmetro com $250 \pm 50$ ovos previamente parasitados, aderido a uma tira de papel de $80 \mathrm{~mm}$ de comprimento por $15 \mathrm{~mm}$ de largura, com três finos filetes de alimento.

Os tubos de emergência com adultos de T. pretiosum com cerca de 24 horas de idade foram conectados às gaiolas de exposição após secagem da calda pulverizada, permitindo a entrada dos insetos no interior da gaiola. Para acelerar a saída dos insetos dos tubos de emergência, foi aumentada a intensidade luminosa da sala durante o período de conexão, que foi de 16 horas. Após esse período, os tubos de emergência foram desconectados e mantidos em condições controladas (temperatura de $25 \pm 1{ }^{\circ} \mathrm{C}$, umidade relativa de $70 \pm 10 \%$ e fotofase de 14 horas) por mais três dias, para que ocorresse a total emergência dos adultos, sendo estes utilizados no cálculo do número de indivíduos que entraram na gaiola. Um sistema de sucção de ar - constituído por bombas de aquário com fluxo invertido e mangueiras - foi conectado às gaiolas de exposição durante todo o período de condução do experimento, evitando o aumento na concentração de gases tóxicos no ambiente interno das gaiolas.

Seis horas após a desconexão dos tubos de emergência, cartões contendo três círculos de $1 \mathrm{~cm}$ de diâmetro com $400 \pm 50$ ovos de A. kuehniella inviabilizados e alimento foram oferecidos às 24 (três cartões), 48 (dois cartões) e 96 (um cartão) horas após pulverização dos pesticidas, a fim de serem parasitados por fêmeas remanescentes de $T$. pretiosum, para avaliação da capacidade de parasitismo, totalizando um período de cerca de 150 horas (aproximadamente seis dias) em que ovos do hospedeiro ficaram disponíveis para o parasitismo.

Sete dias após a aplicação, as gaiolas foram desmontadas e os cartões com ovos do hospedeiro foram delas retirados, transferidos para placas de Petri descartáveis e incubados nas mesmas condições do teste por mais três dias, a fim de que todos os ovos parasitados se tornassem escurecidos, para posteriormente ser realizada a contagem.

O número de fêmeas em cada gaiola foi determinado por contagem do número de ovos parasitados de cada círculo de $1 \mathrm{~cm}$ de diâmetro utilizado na confecção do tubo de emergência, multiplicado pelo número de parasitóides por ovo da população do inseto. O valor obtido foi subtraído do número de adultos que permaneceram no interior do tubo de emergência e multiplicado pela razão sexual da população. Os parâmetros número de parasitóides por ovo e razão sexual foram obtidos mediante avaliação de quatro círculos de $1 \mathrm{~cm}$ de diâmetro contendo ovos parasitados, sendo estes círculos retirados do mesmo cartão usado na confecção dos tubos de emergência.

Com o número de ovos parasitados dividido pelo número de fêmeas que entraram na gaiola, calculou-se o número médio de ovos parasitados por fêmea de $T$. pretiosum de cada tratamento usado para mensurar a capacidade de parasitismo.

As reduções na capacidade de parasitismo dos insetos que tiveram contato com os produtos-teste foram comparadas com a da testemunha (água destilada) e calculadas por meio da fórmula $\mathrm{RP}=\left[1-(P / p)^{*} 100\right]$, em que 
RP é a porcentagem de redução no parasitismo, $P$ é o valor do parasitismo médio para cada produto e $p$ representa o parasitismo médio observado para o tratamento testemunha. Com base nessas porcentagens de reduções no parasitismo, os produtos fitossanitários testados foram classificados, segundo IOBC/ WPRS, em: inócuo (<30\%); levemente nocivo (30-79\%); moderadamente nocivo (80-99\%); e nocivo (>99\%).

Foram utilizadas quatro repetições para cada tratamento, sendo cada gaiola considerada uma unidade experimental no delineamento inteiramente casualizado. Os resultados obtidos referentes à contagem do número de ovos parasitados por fêmea foram testados quanto à normalidade da distribuição dos resíduos, sendo necessária a transformação para vx. Posteriormente, os dados transformados foram submetidos à análise de variação, e as médias dos tratamentos, comparadas pelo teste de Scott \& Knott (Scott \& Knott, 1974) $(\alpha=0,05)$.

\section{RESULTADOS E DISCUSSÃO}

O número médio de ovos parasitados por fêmea de $T$. pretiosum variou significativamente entre os produtos fitossanitários testados (Tabelas 1 e 2). Na testemunha negativa (água destilada), os valores obtidos foram de 33,7 ovos parasitados por fêmea do parasitóide no experimento I (Tabela 1) e de 39,0 ovos parasitados por fêmea no experimento II (Tabela 2). Este parâmetro é um dos critérios mais importantes para validação do teste de seletividade com parasitóides de ovos. A metodologia padronizada pela IOBC/WPRS preconiza para a espécie Trichogramma cacoeciae um número mínimo de 15 ovos parasitados por fêmea (Hassan et al., 2000). Para a espécie $T$. pretiosum ainda não existe esse limiar inferior estabelecido. No entanto, Maceda et al. (2003) estudaram os efeitos da temperatura sobre $T$. pretiosum em ovos de A. kuehniella e observaram, à temperatura de $25^{\circ} \mathrm{C}$, que o número médio de ovos parasitados por fêmea por dia foi de 5,74. Dessa forma, os resultados obtidos no presente trabalho para a testemunha negativa são similares aos observados por esses autores, se for considerado o período de seis dias em que os ovos do hospedeiro A. kuehniella foram expostos às fêmeas para parasitismo.
O inseticida Dipterex ${ }^{\circledR} 500$ reduziu em $100,00 \%$ a capacidade de parasitismo de fêmeas de $T$. pretiosum (Tabelas 1 e 2), confirmando sua toxicidade também para a espécie T. pretiosum. Esse inseticida foi escolhido como testemunha positiva por ter-se revelado nocivo à espécie T. cacoeciae (Grützmacher et al., 2004) na mesma concentração utilizada no presente estudo.

Tabela 1 - Efeito de formulações de glyphosate sobre o número médio $( \pm \mathrm{EP})$ de ovos parasitados por fêmea, redução (\%) na capacidade de parasitismo de Trichogramma pretiosum e classificação de toxicidade desses produtos experimento I. Temperatura: $25 \pm 1{ }^{\circ} \mathrm{C}$; UR: $70 \pm 10 \%$; Fotofase: 14 horas. Pelotas, RS, 2004

\begin{tabular}{|l|c|c|c|c|}
\hline \multicolumn{1}{|c|}{ Produto comercial } & $\mathrm{DC}^{1 /}$ & $\begin{array}{c}\text { Ovos por } \\
\text { fêmea }\end{array}$ & $\mathrm{RP}^{3 /}$ & Classes $^{4 /}$ \\
\hline Testemunha & - & $33,7 \pm 1,81 \mathrm{a}^{2 /}$ & - & - \\
\hline Zapp $^{\mathrm{B}} \mathrm{Qi}$ & 5,8 & $13,5 \pm 1,84 \mathrm{~b}$ & 59,89 & 2 \\
\hline Roundup $^{\mathrm{B}} \mathrm{WG}$ & 4,0 & $13,0 \pm 2,06 \mathrm{~b}$ & 61,56 & 2 \\
\hline Roundup $^{\mathrm{B}}$ (original) & 8,0 & $4,5 \pm 0,20 \mathrm{c}$ & 86,60 & 3 \\
\hline Polaris $^{\mathrm{B}}$ & 8,0 & $4,6 \pm 0,44 \mathrm{c}$ & 86,40 & 3 \\
\hline Dipterex $^{8} 500$ & $300^{*}$ & $0,0 \pm 0,00 \mathrm{~d}$ & 100,00 & 4 \\
\hline
\end{tabular}

I/ $\mathrm{DC}=$ dosagem de campo $\left(\mathrm{kg}\right.$ ou $\mathrm{L}$ do produto comercial $\left.\mathrm{ha}^{-1}\right)$ * $\mathrm{mL} 100 \mathrm{~L}^{-1} ; \stackrel{2}{ }^{\prime}$ Médias acompanhadas pela mesma letra não diferem significativamente pelo teste de Scott \& $\operatorname{Knott~}(\mathrm{P} \leq 0,05) ;{ }^{3 /} \mathrm{RP}=$ redução na capacidade de parasitismo comparado com a testemunha; ${ }^{4 /}$ Classes da IOBC para teste de toxicidade inicial sobre adultos: $1=$ inócuo $(<30 \%) ; 2=$ levemente nocivo $(30-79 \%) ; 3=$ moderadamente nocivo $(80-99 \%) ; 4=$ nocivo $(>99 \%)$.

Tabela 2 - Efeito de formulações de glyphosate sobre o número médio $( \pm \mathrm{EP})$ de ovos parasitados por fêmea, redução (\%) na capacidade de parasitismo de Trichogramma pretiosum e classificação de toxicidade desses produtos experimento II. Temperatura: $25 \pm 1{ }^{\circ} \mathrm{C}$; UR: $70 \pm 10 \%$; Fotofase: 14 horas. Pelotas-RS, 2004

\begin{tabular}{|c|c|c|c|c|}
\hline Produto comercial & $\mathrm{DC}^{-1 /}$ & $\begin{array}{l}\text { Ovos por } \\
\text { fềmea }\end{array}$ & $\mathrm{RP}^{3} /$ & Classes $^{4 /}$ \\
\hline Testemunha & - & $39,00 \pm 3,28 \mathrm{a}$ & - & - \\
\hline $\mathrm{Gliz}^{(1)} 480 \mathrm{CS}$ & 6,0 & $6,33 \pm 1,45 b$ & 83,76 & 3 \\
\hline Glifosato Nortox $^{(B)}$ & 6,0 & $4,61 \pm 2,69 b$ & 88,19 & 3 \\
\hline Glifosato 480 Agripec $^{\circledR}$ & 6,0 & $7,65 \pm 0,65 b$ & 80,40 & 3 \\
\hline Roundup $^{\mathbb{B}}$ Transorb & 6,0 & $5,32 \pm 0,70 \mathrm{~b}$ & 86,42 & 3 \\
\hline Dipterex $^{\circledR} 500$ & $300^{*}$ & $0,00 \pm 0,00 \mathrm{c}$ & 100,0 & 4 \\
\hline
\end{tabular}

$1 / \mathrm{DC}=$ dosagem de campo $\left(\mathrm{kg}\right.$ ou $\mathrm{L}$ do produto comercial $\left.\mathrm{ha}^{-1}\right)$ * mL 100L $\mathrm{L}^{-1} ; \stackrel{2 / /}{ }$ Médias acompanhadas pela mesma letra não diferem significativamente pelo teste de Scott \& Knott $(\mathrm{P} \leq 0,05)$; $^{3 /} \mathrm{RP}=$ redução na capacidade de parasitismo comparado com a testemunha; 4/ Classes da IOBC para teste de toxicidade inicial sobre adultos: $1=$ inócuo $(<30 \%) ; 2=$ levemente nocivo $(30-79 \%) ; 3=$ moderadamente nocivo $(80-99 \%) ; 4=$ nocivo $(>99 \%)$ 
Todas as formulações de glyphosate testadas afetaram a capacidade de parasitismo de fêmeas de $T$. pretiosum, porém com reduções no parasitismo variando de 59,89 a $88,19 \%$. No entanto, $75,00 \%$ das formulações testadas reduziram o parasitismo acima de $80,00 \%$, sendo moderadamente nocivas (classe 3) aos adultos de T. pretiosum (Tabelas 1 e 2).

Os herbicidas Zapp ${ }^{\circledR}$ Qi e Roundup ${ }^{\circledR}$ WG foram levemente nocivos, sendo categorizados na classe 2 , com reduções no parasitismo das fêmeas de $T$. pretiosum variando de 59,89 a $61,56 \%$, respectivamente (Tabela 1 ).

Os herbicidas Roundup ${ }^{\circledR}$, Polaris ${ }^{\circledR}$, Gliz $^{\circledR}$ 480 CS, Glifosato Nortox $^{\circledR}$, Glifosato 480 Agripec $^{\circledR}$ e Roundup Transorb ${ }^{\circledR}$ apresentaram redução no parasitismo variando de 80,40 a $88,19 \%$ (Tabelas 1 e 2), sendo moderadamente nocivos (classe 3) aos parasitóides. Trabalhando com T. cacoeciae e o herbicida Roundup ${ }^{\circledR}$ na concentração de $0,36 \%$ de equivalente ácido, Hassan et al. (1988) classificaram este herbicida como levemente nocivo (classe 2). No presente estudo, a concentração de equivalente ácido para Roundup foi de $14,4 \mathrm{mg} \mathrm{L}^{-1}$, correspondente a $1,44 \%$; portanto, bem superior à que foi avaliada pelos autores, demonstrando incremento na toxicidade do herbicida com o aumento da concentração na calda de aplicação.

Analisando os resultados apresentados nas Tabelas 1 e 2, observa-se que as duas formulações comerciais de glyphosate levemente nocivas (classe 2) foram Zapp ${ }^{\circledR}$ Qi e Roundup ${ }^{\circledR}$ WG. Essas formulações apresentam em suas composições sais potássico e amônio, respectivamente, e diferem das demais, que foram moderadamente nocivas (classe 3) e apresentaram, ligado ao ingrediente ativo, sal de isopropilamina. Esses resultados propiciam indícios de que o tipo de sal presente na formulação do glyphosate se constitui em fator preponderante para a toxicidade à forma adulta do parasitóide T. pretiosum. Avaliando a seletividade de diferentes formulações de glyphosate a estirpes de Bradyrhizobium, Santos et al. (2004) verificaram que a única formulação que continha sal potássico (Zapp ${ }^{\circledR}$ Qi) apresentou menor toxicidade a todas as estirpes testadas, sendo inclusive não-tóxica para uma determinada estirpe.
Testes de toxicidade em laboratório submetem os insetos-teste a uma exposição máxima aos resíduos dos produtos fitossanitários testados e constituem a primeira etapa da seqüência de testes preconizada pela IOBC/WPRS (Hassan et al., 2000; Hassan \& Abdelgader, 2001).

De acordo com os resultados obtidos, as formulações dos herbicidas Zapp ${ }^{\circledR}$ Qi e Roundup ${ }^{\circledR}$ WG foram seletivas em favor do parasitóide de ovos T. pretiosum e, conforme a seqüência da IOBC/WPRS, não serão testadas nas etapas subseqüentes. Para as formulações comerciais Roundup $^{\circledR}$, Polaris ${ }^{\circledR}$, Gliz $^{\circledR} 480$ CS, Glifosato Nortox $^{\circledR}$, Glifosato 480 Agripec $^{\circledR}$ e Roundup ${ }^{\circledR}$ Transorb, que foram moderadamente nocivas (classe 3), os resultados obtidos não devem ser extrapolados para condições de campo e deverão avançar na seqüência de testes da IOBC/ WPRS, sendo avaliados em testes a campo, em condições específicas de uma determinada cultura e sistema de manejo. Outras formulações comerciais também deverão ser estudadas, a fim de que os agricultores possam optar, no momento da aquisição do herbicida, por moléculas seletivas aos inimigos naturais.

\section{LITERATURA CITADA}

AMARANTE JÚNIOR, O. P. et al. Glifosato: propriedades, toxicidade, usos e legislação. Química Nova, v. 25 , p. $589-593,2002$.

GRÜTZMACHER, A. D. et al. The side-effects of pesticides used in integrated production of peaches in Brazil on the egg parasitoid Trichogramma cacoeciae Marchal (Hym., Trichogrammatidae). J. Appl. Entomol., v. 128, p. 377-383, 2004.

HARTZLER, B. Which glyphosate product is best?. Disponível em: <http:www.weeds.iastate.edu/mgmt/ qtr01-1/glyphosateformulations.htm $>$. Acesso em: 28 jan. 2001.

HASSAN, S. A.; ABDELGADER, H. A sequential testing program to assess the effects of pesticides on Trichogramma cacoeciae Marchal (Hym., Trichogrammatidae). IOBC/WPRS Bulletin, v. 24, p. 71-81, 2001.

HASSAN, S. A. et al. Results of the fourth joint pesticide testing programme carried out by the IOBC/WPRS-Working group"pesticides and beneficial organisms". J. Appl.

Entomol., v. 105, p. 321-329, 1988.

Planta Daninha, Viçosa-MG, v. 23, n. 3, p. 457-462, 2005 
HASSAN, S. A. et al. A laboratory method to evaluate the side effects of plant protection products on Trichogramma cacoeciae Marchal (Hym., Trichogrammatidae). In: CANDOLFI, M. P. et al. (Eds.) Guidelines to evaluate side-effects of plant protection products to non-target arthropods. Reinheim: IOBC/ WPRS, 2000. p. 107-119.

HEAP, I. M. The occurrence of herbicide resistant weeds world wide. Pest. Sci., v. 51, p. 235-243, 1997.

MACEDA, A.; HOHMANN, C. L.; SANTOS, H. R. Temperature effects on Trichogramma pretiosum Riley and Trichogrammatoidea annulata De Santis. Braz. Arch. Biol. Techonol., v. 46, n. 1, p. 27-32, 2003.

PARRA, J. R. P. Técnicas de criação de Anagasta kuehniella, hospedeiro alternativo para produção de Trichogramma. In: PARRA, J. R. P.; ZUCCHI, R. A. (Eds.). Trichogramma e o controle biológico aplicado. Piracicaba: FEALQ, 1997. p. 121-150.

PARRA, J. R. P.; ZUCCHI, R. A. Trichogramma no Brasil: viabilidade de uso após vinte anos de pesquisa. Neotr. Entomol., v. 33, p. 271-282, 2004.
SANTOS, J. B. et al. Efeitos de diferentes formulações comerciais de glyphosate sobre estirpes de Bradyrhizobium. Planta Daninha, v. 22, p. 293-299, 2004.

SCOTT, A. J.; KNOTT, M. A cluster analysis method for grouping means in the analysis of variance. Biometrics, v. 30, p. $507-512,1974$.

SHANER, D. L. The impact of glyphosate-tolerant crops on the use of other herbicides and on resistance management. Pest Manag. Sci., v. 56, p. 320-326, 2000.

STEIN, C. P.; PARRA, J. R. P. Uso da radiação ultravioleta para inviabilizar ovos de Anagasta kuehniella (Zeller, 1879) visando estudos com Trichogramma spp. An. Soc. Entomol. Brasil, v. 16, n. 1, p. 229-233, 1987.

ZUCCHI, R. A.; MONTEIRO, R. C. O gênero Trichogramma na América do Sul. In: PARRA, J. R. P.; ZUCCHI, R. A. (Eds.) Trichogramma e o controle biológico aplicado. Piracicaba: FEALQ, 1997. p. 41-46. 